\title{
EI sistema tutorial: una alternativa para la gerencia académica en La Universidad del Zulia
}

Luz Maria Martínez de Correa*

\section{Resumen}

Este trabajo tiene por finalidad presentar algunas estrategias para la implantación de un "Sistema Tutorial". Para ello, se abordan tres cuestiones: la primera, intenta presentar un diagnóstico situacional atendiendo a los problemas que se le presentan a muchos participantes en la etapa de culminación de sus estudios de Postgrado, referido al desarrollo del Trabajo de Grado, la segunda consiste en ilustrar la imagen objetivo del tutor, y por último presenta una propuesta de Programa para un Sistema Tutorial, la cual introduce una modalidad de evaluación para la gerencia académica, en función de la efectividad y eficacia del Trabajo de Grado.

Palabras claves: Sistema Tutorial, Tesis de Grado, Evaluación del Docente.

\section{The Tutorial system, an alternative to academic management at the University of Zulia}

\section{Abstract}

This paper introduces some strategies for implementation of a Tutorial System. In order to do this, three main aspects are dealt with: first, a diagnosis of students' problems while in their Master theses process at the end of their graduate studies Second, a description of desired graduate Advisor's role or Advisor objetive image. Third, a suggested program for a Tutorial System. This System introduces strategies for Academic

Recibido: 24-09-96 - Aceptado: 06-12-96

* Investigadora del Instituto de Filosofía del Derecho. "Dr. J.M.Delgado Ocando". Jefa de la Sección de Sociología Jurídica. Facultad de Ciencias Juridicas y Politicas Universidad del Zulia. Fax: 061-430117-596657. E-mail Imartine@europa.ica.luz.ve 
Management Assessment taking into consideration the effectiveness and efficiency of Master theses produced under advisor's tutorial work.

Key words: Tutelage Sistem, Master Thesis

\section{Introducción}

La idea de diseñar este programa denominado "Sistema Tutorial", subyace en la experiencia de la docencia durante década y media, en la cual se observa con gran preocupación que en las universidades se enseña, se orienta, pero poco se evalúa la acción tutorial y por ende no se han alcanzado los niveles deseables en materia de investigación, siendo ésta la que conduce a nuevos caminos, y perspectivas para la clencia y la intervención científica.

Esta dificil realidad, es alarmante debido a que más del $50 \%$ de los docentes universitarios, no están conscientes de la gran responsabilidad que tienen como conductores de procesos educativos y de investigación, en muchos casos el docente se cree dueño y señor del saber, por consiguiente, teme ser evaluado, $y$ en muchos casos, considera que en el proceso evaluativo en la práctica universitaria no se adoptan los preceptos que consagra el Reglamento Universitario en materia evaluativa. Durante mucho tiempo se ha tenido la creencia que la evaluación es un acto punitivo y no un sistema de control para la calidad docente, la evaluación incluye una gran variedad de evidencias necesarias para mejorar la actividad tutorial, así como también el crecimiento personal en docencia e investigación.

A la luz de este problema, se puede apreciar que la dimensión individual del tutoriado juega un papel determinate en el éxito o fracaso del proceso, es por ello que la actuación de ambos sujetos (tutortutoriado), influye de forma determinante en el proceso. En muchos casos, el tesista concibe el Trabajo de Grado (T.G.), como: "una carga obligatoria", a la que debe dar cumplimiento en un tiempo determinado, de no ser asi, no pueden ver cristalizados sus esfuerzos, ni mucho menos cumplir con el requisito de grado.

Aunque en la actualidad, se ha creado más conciencia acerca del significado que tiene el Trabajo de Grado, se evidencia en la mayoría de los Programas de Postgrado un gran número de participantes que no logran realizar su Trabajo de Grado, en tal sentido, la actitud del tesista y las tareas que hace en relación a su T.G, en muchos casos se consideran como intrínsecamente extrañas a él, que en todo caso se le pueden denominar como el síndrome del T.M.T (todo menos tesis); surgen entonces incertidumbres que se traducen en preguntas, tales como: ¿por qué ocurre esto?. Para responder a esta interrogante, se puede afirmar de forma categórica, que en la mayoría de los casos, estos problemas obedecen a la ausencia de un Sistema Tutorial en el proceso de conducción del Trabajo de Grado. Por tal motivo, los tesistas se sienten desprovistos de un guía que les facilite en forma eficaz y eficiente la dificil tarea del llamado Trabajo de Grado. 
Aún cuando existen en algunas universidades, coordinaciones de seminarios de grado, direcciones de tesis, entre otros, también existen otros problemas colaterales, entre los cuales se destacan: la falta de supervisión del sistema académico, la actitud paternalista o anárquica del tutor, una formación deficiente (contenido y metodología), inexperiencia en investigación, la no presencia de los preceptos pedagógicos que se traducen en poca o nula comunicación, por todo lo antes dicho, se hace necesario un sistema que brinde la posibilidad de realizar un proceso que garantice el cumplimiento eficiente y eficaz del Trabajo de Grado.

El propósito de presentar este trabajo sobre el "Sistema Tutorial", consiste en introducir en la colectividad cientifica tres cuestiones:

* La primera, intenta presentar un diagnóstico situacional en lo atinente a los problemas que se le presentan a muchos participantes en el proceso de desarrollo del Trabajo de Grado.

* La segunda consiste en ilustrar la imagen objetivo del Tutor.

* La tercera, propone un Programa para un Sistema Tutorial que muestra: la organización del mismo, que incluye la cadena de actores involucrados en el proceso (coordinadores, directores, tutores y participantes) y las distintas modalidades de evaluar la acción tutorial.

La acción tutorial recae en el tutor, porque es él, quién tiene el timón en la mano y está llamado a conducir un proceso que implica: dedicación, responsabilidad, creatividad, dominio cognoscitj- vo, dominio metodológico y planeamiento de sus funciones.

Esta obra intenta poseer un carácter didáctico, debido a que presenta una estructura semántica de fácil comprensión y una condición práctica en los instrumentos de medición y recomendaciones propuestas; no pretende ser la panacea a los problemas planteados, sino una publicación de carácter divulgativo y didáctico que contribuya a dar un beneficio para sus lectores.

\section{Previos Teóricos}

La formación intelectual del hombre nunca concluye, el ejercicio de aprender en sus diferentes modalidades está siempre presente en el ser humano, y como tal, es el hombre en su afán de ampliar nuevos horizontes, el principal consumidor de los conocimientos que aportan la ciencia la tecnología y la cultura.

Desde este punto de vista, la prosecución académica de cientos de profesionales universitarios adquiere significado, en la medida que éstos son capaces de culminar una meta científica. De igual manera adquiere sentido la noble competencia de las diferentes universidades del mundo, las cuales ofrecen programas de postgrado bajo criterios específicos de indagar y profundizar conocimientos científicos a través de la investigación.

En Venezuela la efervescencia por el perfeccionamiento académico, además de estar consustanciada a la atmósfera envolvente de un siglo que bien puede definirse como agresivo, creativo $y$ progresista, las universidades no escapan de esta ineludible realidad. Otro as- 
pecto que llama la atención, es la presencia de un crecimiento acelerado de nuevas universidades y la diversidad de ofertas académicas a nivel de Postgrado.

En ambos niveles, la figura del Tutor del Trabajo de Grado es relevante y su acción tutorial debe centrarse en el tesista, sin embargo "Ia educación recibida no proporciona el instrumental preciso para enfrentar esa tarea..." (Sabino, 1987:7), es por ello que el autor plantea que es frecuente que docentes, investigadores y profesionales en general padezcan de los mismos problemas.

Por igual ruta anda quién expresa con similar inquietud:

Se dedican muchos esfuerzos para atender debidamente a los participantes mientras están recibiendo los cursos regulares de sus carreras, pero cuando concluyen su escolaridad quedan un poco "a la mano de Dios", sin recibir toda orientación y ayuda necesaria para poder realizar sus trabajos finales de graduación. (Brenes, 1987:7)

Ante tan manifiesta y sensible preocupación, extraídas de entre las muchas que existen sobre la realidad y prospección de los Trabajos Especiales de Grado, al profundizar sobre las posibles causas incidentes $y$, desde luego sobre los efectos de las mismas, éstas revisten una especial atención en lo relativo: a) "el nivel de formación del tutor" y b) la "relación con el proceso metódico y pedagógico en la acción tutorial", pues con la ausencia de uno o de ambos elementos, serian más daños causados al tesista que la pretendida intención de bondad implicada en la acción tutorial.

Referirse al nivel de Formación del tutor, luce como sin sentido, por cuanto se supone que el mismo debe ser poseedor de una excelente preparación académica, esta suposición, no carente de valor y aceptación unánime, amerita ser analizada en profundo, ya que si se toma la realidad dicotomizada del homo sapiens en cuanto a su psiquis e intelecto se refiere, puede partirse del principio de que no todo aquél que tenga formación puede tener mucho de instrucción. Se aclara, que el ser humano, en su intelecto - en su espiritu puede ser un "ser preparado", sin que se produzca la integración de ambas realidades en una situación que se perciba de una manera precisa y concreta.

El balance entre el aspecto cognitivo, afectivo y la acción misma del proceso, pareciera estar sobreentendido en el término formación. Piénsese, entonces que es necesaria la formación del tutor, como parte del cultivo de su patrimonio intelectual, sin que ello implique descuido de las energías que deben estar en movimiento.

\footnotetext{
"la acción es claramente más significativamente que la percepción" por cuanto "los seres humanos se relacionan entre si en términos de acciones", y éstas, "...son los sucesos de mayor importancia y también los que pueden observarse y medirse mejor". (Sears, $R$, citado por Parsons y Shils, 1962: 525).
}

La experiencia académica universitaria venezolana, es a diario silente testi- 
go de un buen número de profesores cargados de conocimientos, pero en atgunnos casos vacíos de sensibilidad y creatividad, elementos indispensables para involucrar con ahínco al tesista en el exigente quehacer investigativo. En este caso, adquiere sentido la afirmación de Sears cuando señala que "la acción no tiene lugar en un organismo psicológicamente vacío". (Citado por Parsons y Shils, 1962:525).

De igual forma, la experiencia académica universitaria es víctima de una extremada bondad profesoral manifestada en concesiones generosas, que afectan, hasta cierto punto, el epíteto universitario. Sería entonces, la formación integral del tutor, la que decida si él, se encuentra lo suficientemente apto para servir de asesor y de punto de apoyo al tesista, y to necesariamente humano como para administrar con prudencia y cálculo situaciones de comprensión y ayuda, que, en lugar de desfavorecer el esfuerzo por la búsqueda de la verdad científica, lo estimulen y refuercen aún en las condiciones más adversas.

Es conveniente destacar, que si bien se evidencia una fuerte tendencia a pensar en el tutor como dueño y señor de una parcela del conocimiento, pero éste no es muchas veces, lo más importante en la acción tutorial, por cuanto el tutor está frente a estructuras humanas complejas y diferentes entre sí, para las cuales deberá generar apoyo y orientación similar. Estas estructuras humanas complejas no siempre operan en la misma línea, ni codifican y descodifican con la misma facilidad, claridad e intensidad las orientaciones recibidas. Por tanto, un ex- celente tutor estaria atento a esas individualidades y adecuaría sus mensajes a tales realidades.

Vista de esta manera, la acción tutorial no se desliga de la indispensable y necesaria preparación del mismo. Se encamina hacia la integración con el hombre, pensando éste como persona y centro de acciones que tienden al encuentro con la verdad, por ello el tutor:

"...mira el progreso del individuo y abre camino a una sociología aplicada, capaz de instaurar un orden nuevo (Carrier, H., 1977:10).

Estas reflexiones, en torno a la formación del tutor de "Trabajo de Grado", conllevan a descubrir la presencia de variables intervinientes, que ligadas a las circunstancias de la acción tutorial, hacen pensar, en dos polaridades: quien tutoriza y el tutoriado. Ambos son producto de inquietudes diversas, y en algunos casos de gran similitud.

El elemento unitario de las diferencias individuales, radica en la preocupación que presenta para el tesista resolver el problema referente al contenido de su Trabajo de Grado, y en la capacidad y deseo del tutor de ayudar y dar apoyo a la investigación que se emprenda. Queda establecido con claridad, que el tutor debe poseer una formación integral para garantizar una acción tutorial plena.

\section{Situación problema}

* En Venezuela las universidades, pueden incorporar un sistema tutorial acorde con los preceptos y disposiciones contenidas en la Ley de Universidades. 
"Ias UnIversidades deben reallzar una función rectora en la educación, la cultura y la clencla. Para cumpilir esta mis/ón, sus actlvidades se diriglrón a crear, aslmilar y difundir el saber medlante la Investigación y la ensefianza, a completar la formación Integral Iniclada en los clclos educaclonales anterlores y formar los equipos profeslonales y técnlcos que neceslta la Naclón para su desarrollo y progreso". (Ley de Universidades, art. 39).

\section{En La Universidad del Zulia (LUZ)}

Existe ausencia de un Sistema de Supervisión Institucional en el proceso del Trabajo de Grado, debido a que no se cuenta con un Sistema Tutorial que responda al papel que debe cumplir el tutor para actuar con eficiencia en las etapas del proceso que dirige a nivel de postgrado.

- La creación de Centros de Investigación y el incremento de Programas de Maestrias, ha permitido, por una parte, la conformación de una comunidad académica altamente motivada que hoy comparte un conjunto básico de conceptos y criterios científicos acerca del rol que le corresponde al tutor y la consiguiente producción de investigación.

\section{Solución al problema}

- Se recomienda dictar un Taller de Gerencia Tutorial para los profesores que conformarán la plataforma tutorial, en los respectivos Programas de Maestrías se propone un Sistema Tutorial con dos componentes fundamentales: Seminario 1 y Seminario II con ( 3 unidades créditos cada uno). Es de hacer notar que en LUZ existen post-grados con nivel de especialización, maestría y doctorado, en este trabajo nos referimos al nivel de maestría.

* El Seminario l: Trata de la identificación de un problema, su construcción teórica o histórica a los fines de la investigación; proporcionando los recursos metodológicos y técnicos, así como las proposiciones analíticas para abordar un tema en particular.

La asignatura se inscribe en un programa de investigación dirigido por uno o más docentes (con un coordinador), sobre una temática en concreto, de interés para el Programa de Postgrado, a partir del cual se determinarán líneas de investigación a ser desarrolladas por el participante $y$ el profesor coordinador de un proyecto de investigación. Abscrito a los Centros, Institutos y otras unidades de investigación de la universidad.

* Seminario II: Es la continuidad de la investigación iniciada en el Seminario de Grado I, culminando con la presentación de un Trabajo de Grado. En este nivel el profesor actuará como asesor-guía, obteniendo los participantes una mayor autonomía en el desarrollo de la investigación.

\section{Propósito general del sistema}

1. Fomentar el espiritu científico, estimulando el interés por la investigación y cultivando el hábi- 
to del razonamiento, la crítica, la adecuada exposición del pensamiento escrito y la honestidad intelectual.

2. Orientar hacia la especialización profesional, proporcionando un conocimiento y manejo de las corrientes teóricas y recursos de Investigaciones desarrolladas en el estudio de un campo específico de la realidad.

3. Revisar la Estructura Organizativa de los datos.

4. Corregir cada versión del trabajo escrito por tesista.

5. Asesorar para la presentación del T.G.

6. Revisar y evaluar el Trabajo de Grado en General.

7. Enseñar a los tesistas los diferentes medios instruccionales para la presentación y defensa del Trabajo.

El coordinador del Seminario velará por el cumplimiento de las actividades programadas en este sistema tutorial, tanto para el Seminario I como el Seminario II, el tutor llevará continuidad en ambas fases del proceso de tutoría y el asesoramiento respectivo del Trabajo de Grado.

\section{Selección de los Tutores}

En cuanto a la selección de los docentes para dirigir los Seminarios, éstos deben cumplir con los requisitos para tal fin.

En el cuadro No. 1 se representa la "IMAGEN OBJETIVO" del Perfil ideal del Tutor. La Planificación Estratégica, denomina Imagen Objetivo al nivel ópti- mo deseado, en términos de eficacia, debido a que ésta representa el grado en que se alcanzan los objetivos y metas de acuerdo a un tiempo determinado, independientemente de los costos que implique. La eficiencia se asocia a la optimización de los recursos para maximizar el producto (T.G), que redundará en productividad científica.

En cualquiera de los casos, no es obligatorio que las (24 cualidades) presentadas, sean el requisito último de selección del tutor, es necesario que el profesor posea un $50 \%$ de ellas para fijar los criterios de selección.

Para la selección del tutor en los Seminarios I y II se debe preparar un banco de información profesoral que elaborará el Coordinador de Seminario, conteniendo las caracteristicas y niveles deseados del futuro tutor, a fin de lograr un nivel elevado de eficacia del Sistema.

\section{Objetivos del Seminario I}

El Programa del Sistema Tutorial que se presenta está dirigido a los profesores, directores, $y$ tutores de los Seminarios I y II, a fin de que éstos cumplan con las etapas y estrategias diseñadas para cada seminario.

Esta primera fase, exige una serie de condiciones académicas y pedagógicas a objeto de indicarle al tutor, los componentes que se necesitan para el asesoramiento y supervisión, ella ofrece criterios metodológicos y temáticos para la acción tutorial.

Objetivos

Elaborar líneas de Investigación 


\section{Cuadro $N^{2} 1$ \\ Imagen objetivo del tutor}

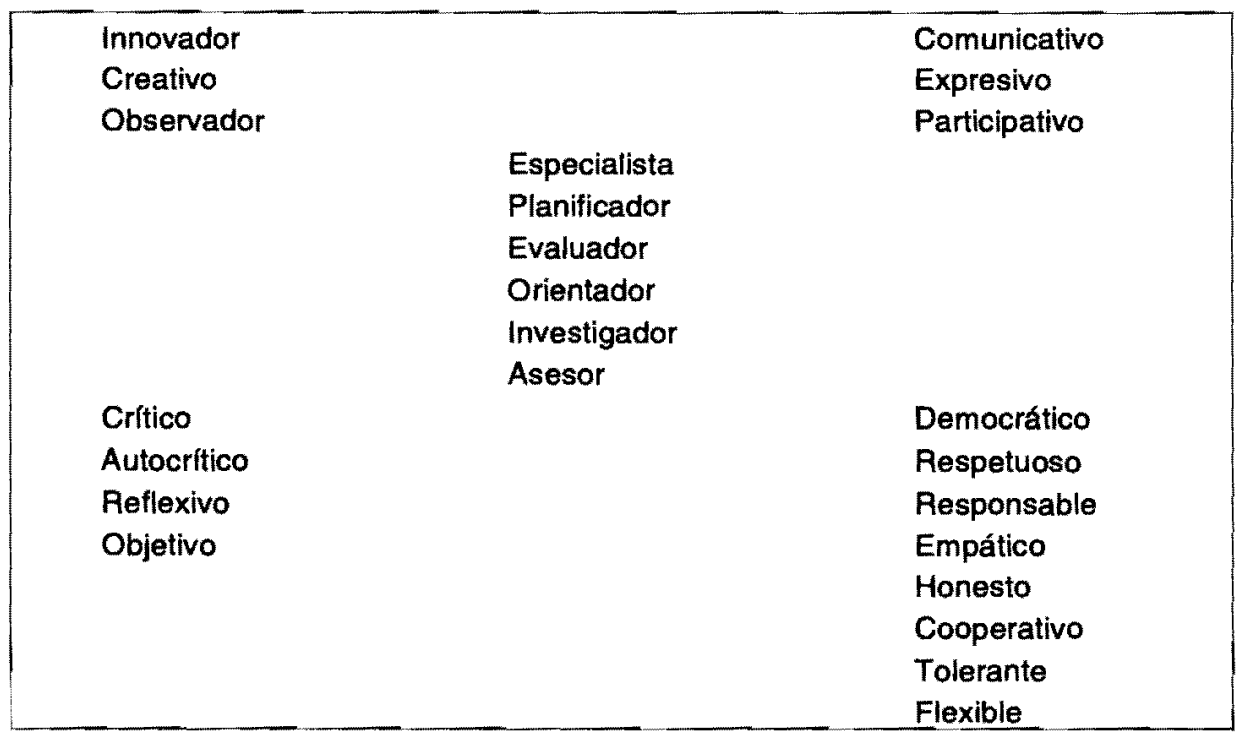

- Planificar actividades con los Tesistas

- Orientación Metodológica

- Orientación Teórica

- Seguimiento de los enfoques teóricos y metodológicos

- Evaluación y Control

Ver Cuadro No. 2

\section{Objetivos del Seminario II}

Una vez cublerta la fase del Seminario $\mathrm{l}$, el profesor:

- Debe orientar, enseñar y evaluar en cada momento del seminario.

- Orientar sobre el diseño concreto de Investigación.

- Asesorar y revisar los instrumentos y técnicas a utilizar.

Ver Cuadro No. 3

\section{Sistema de información}

Este aspecto alude al reconocimiento de un sistema que pretende mostrar elementos substanciales que le dan coherencia a las fases que contiene el mismo. Entendiendo que el Sistema de Información es un proceso que articula hechos y generalizaciones relacionadas coherentemente, dentro de un particular campo de trabajo o estudio, asi, por ejemplo, se espera que un químico haya aprendido y almacenado un cuerpo espe. cializado de conocimientos sobre la química.

En resumen, es evidente que pueden identificarse algunas razones por las que debe aprehenderse la información, 
Cuadro $\mathrm{N}^{2} 2$

Plan operativo por objetivos (Seminario I)

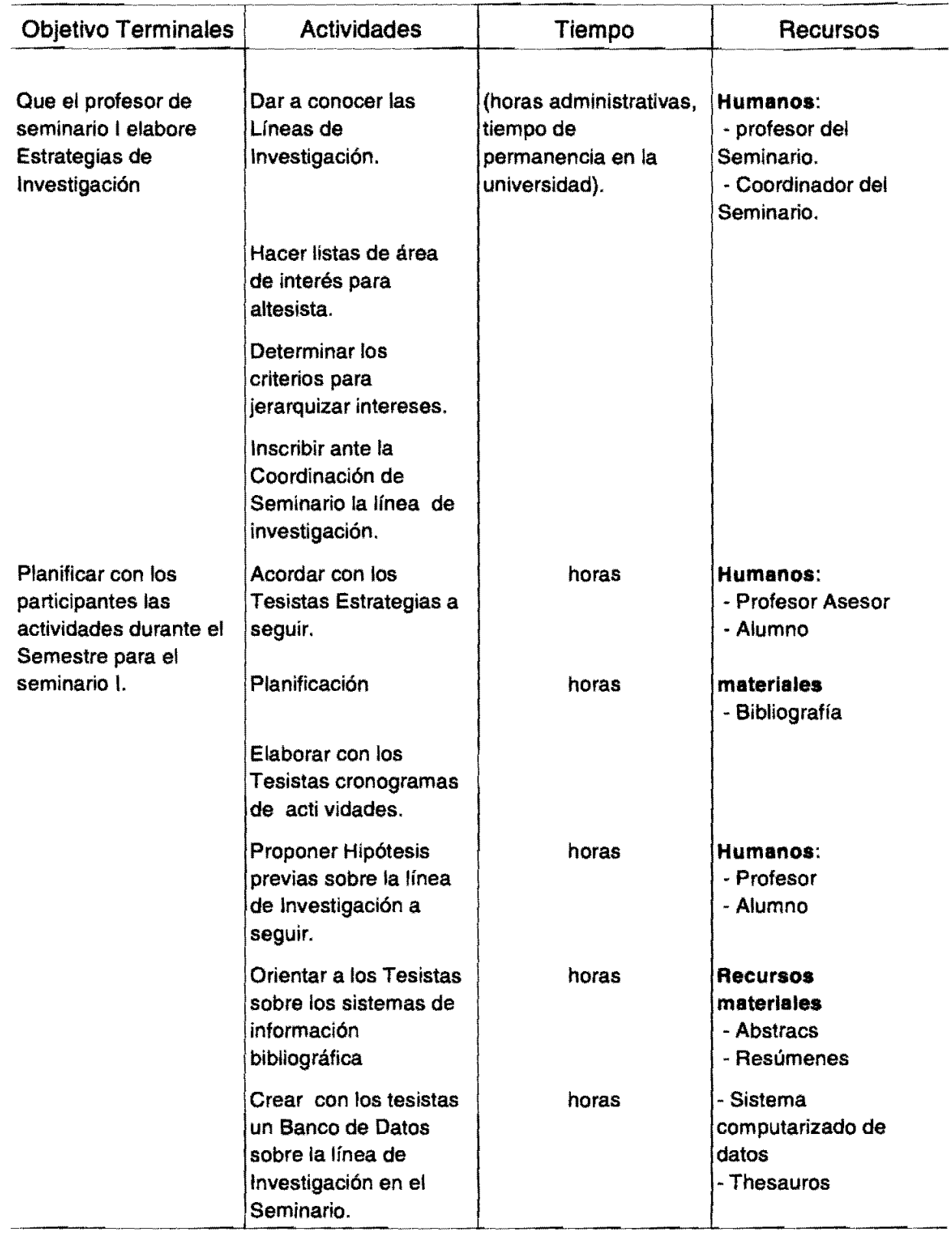




\section{Cuadro № 2}

Plan operativo por objetivos (Seminario I) cont...

\begin{tabular}{|c|c|c|c|}
\hline Objetivo terminales & Actividades & Tiempo & Recursos \\
\hline \multirow[t]{2}{*}{$\begin{array}{l}\text { Llevar un seguimiento } \\
\text { de las teorias } \\
\text { utilizadas por los } \\
\text { tesistas. }\end{array}$} & $\begin{array}{l}\text { Exigir a los tesistas un } \\
\text { reporte de las tearias. }\end{array}$ & horas & $\begin{array}{l}\text { Humanos: } \\
\text { - Protesor } \\
\text { - Alumno }\end{array}$ \\
\hline & $\begin{array}{l}\text { Orientar a los tesistas } \\
\text { para la realización de } \\
\text { Descriptores del Tema } \\
\text { objeto de Estudio. }\end{array}$ & horas & $\begin{array}{l}\text { Recursos materiales } \\
\text {-Bibliografia }\end{array}$ \\
\hline \multirow[t]{6}{*}{$\begin{array}{l}\text { Presentar los } \\
\text { diferentes tipos de } \\
\text { metodologías a utilizar }\end{array}$} & $\begin{array}{l}\text { Revisar con los } \\
\text { Tesistas la } \\
\text { metodología autilizar. } \\
\text { Revisar áreas } \\
\text { problemas de interés. }\end{array}$ & horas & \\
\hline & $\begin{array}{l}\text { Determinar con el } \\
\text { tesista el problema, } \\
\text { preguntas o hipótesis } \\
\text { de investigación. }\end{array}$ & horas & $\begin{array}{l}\text { Humanos: } \\
\text { - Protesor } \\
\text { - Alumno }\end{array}$ \\
\hline & $\begin{array}{l}\text { Señalar los criterios } \\
\text { para la elaboración } \\
\text { del Planteamiento del } \\
\text { problema. }\end{array}$ & horas & \\
\hline & $\begin{array}{l}\text { Revisar la primera } \\
\text { versión. }\end{array}$ & horas & \\
\hline & $\begin{array}{l}\text { Revisión segundo } \\
\text { borrador del } \\
\text { Planteamiento del } \\
\text { problema. }\end{array}$ & horas & \\
\hline & $\begin{array}{l}\text { Revisar el enfoque } \\
\text { metodológico y su } \\
\text { articulación con la } \\
\text { primera vesrsión. }\end{array}$ & horas & \\
\hline $\begin{array}{l}\text { Determinar con los } \\
\text { Tesistas los previos } \\
\text { teóricos. }\end{array}$ & $\begin{array}{l}\text { Exigir la primera } \\
\text { versión de los previos } \\
\text { teóricos. Corregir } \\
\text { enfoques teóricos. }\end{array}$ & horas & $\begin{array}{l}\text { - Protesor } \\
\text { - Alumno }\end{array}$ \\
\hline
\end{tabular}




\section{Cuadro $N^{\circ} 2$}

Plan operativo por objetivo (Seminario I) cont...

\begin{tabular}{|c|c|c|c|}
\hline Objetivo terminales & Actividades & Tiempo & Recursos \\
\hline $\begin{array}{l}\text { Entregar a la } \\
\text { Coordinación un } \\
\text { informe detallado } \\
\text { de la actuación del } \\
\text { tesista. }\end{array}$ & $\begin{array}{l}\text { Exigir segundo } \\
\text { bo- rrador. } \\
\text { Evaluar todo el pro- } \\
\text { ceso desde la Fase } \\
\text { del problema al } \\
\text { - marco teórico. } \\
\text { Entregar un reporte } \\
\text { a cada estudiante } \\
\text { sobre su actuación } \\
\text { con los siguientes } \\
\text { criterios: } \\
\text { - Responsabilidad } \\
\text { - Nivel de análisis y } \\
\text { reflexión teórica. } \\
\text { Coevaluación } \\
\text { Profesor Alumno } \\
\text { Autoevaluación } \\
\text { Tesista Profesor. } \\
\text { Discutir con el } \\
\text { Coornador los } \\
\text { resultados del } \\
\text { Seminario. }\end{array}$ & $\begin{array}{l}\text { horas } \\
\text { horas } \\
\text { (horas de } \\
\text { permanencia en la } \\
\text { universidad). }\end{array}$ & $\begin{array}{l}\text { - Profesor } \\
\text { - Alumno } \\
\text { - Coordinador } \\
\text { Recursos } \\
\text { materiales } \\
\text { - Tablas de } \\
\text { evaluación } \\
\text { - Instrumentos de } \\
\text { Evaluación }\end{array}$ \\
\hline
\end{tabular}




\section{Cuadro № 3 \\ Plan operativo por objetivos}

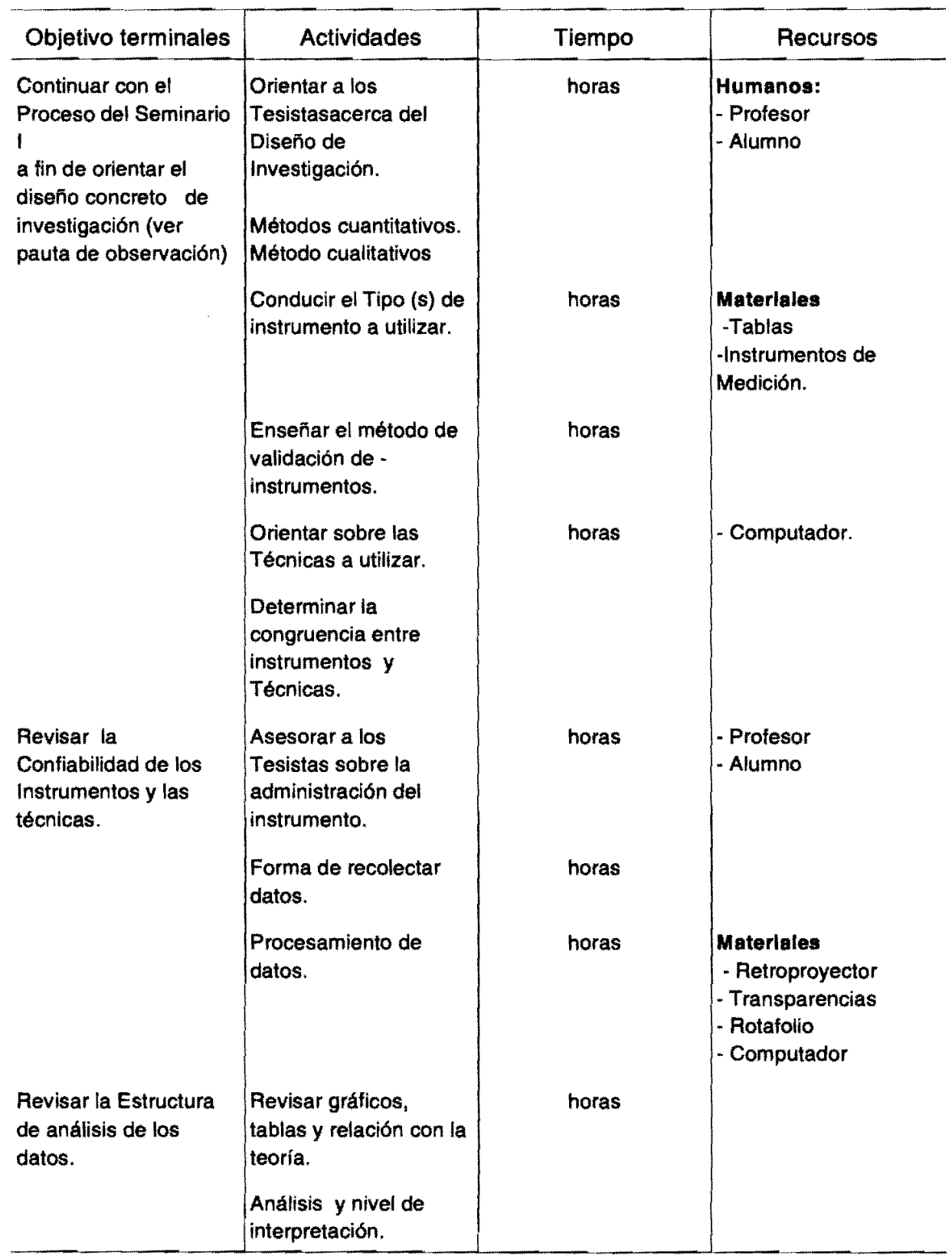


Cuadro № 3

Plan operativo por objetivos (Seminario II) cont...

\begin{tabular}{|c|c|c|c|}
\hline Objetivo Terminales & Actividades & Tiempo & Recursos \\
\hline & $\begin{array}{l}\text { Orientar sobre la } \\
\text { sistematización de } \\
\text { los capitulos Plan } \\
\text { de Tesis). }\end{array}$ & horas & \\
\hline & $\begin{array}{l}\text { Revisar el primer } \\
\text { capítulo. }\end{array}$ & horas & $\begin{array}{l}\text { - Profesor } \\
\text { - Alumno }\end{array}$ \\
\hline & $\begin{array}{l}\text { Exigir congruencia } \\
\text { entre objetivos, } \\
\text { Previos teóricos y } \\
\text { resultados. Revisar } \\
\text { los sucesivos } \\
\text { capítulos. }\end{array}$ & horas & $\begin{array}{l}\text { Materiales } \\
\text {-Tabla de evaluación }\end{array}$ \\
\hline & $\begin{array}{l}\text { Evaluar y } \\
\text { corregir los } \\
\text { capítulos } \\
\text { respectivos. }\end{array}$ & horas & \\
\hline $\begin{array}{l}\text { Corregir la Primera } \\
\text { versión del trabajo } \\
\text { en forma general. }\end{array}$ & $\begin{array}{l}\text { Realizar con los } \\
\text { Tesistas un estudio } \\
\text { de congruencia }\end{array}$ & horas & $\begin{array}{l}\text { Materiales } \\
\text { - Instructivo para } \\
\text { evaluarel T.G. } \\
\text { - (Pauta de } \\
\text { Observación) }\end{array}$ \\
\hline $\begin{array}{l}\text { Asesorar a los } \\
\text { tesistas en la } \\
\text { presentación del } \\
\text { Trabajo Especial } \\
\text { de Grado. }\end{array}$ & $\begin{array}{l}\text { Enseñar las } \\
\text { Estrategias } \\
\text { Metodológicas para } \\
\text { la Realización de la } \\
\text { Introducción y } \\
\text { Resumén y } \\
\text { conclusiones. }\end{array}$ & horas & $\begin{array}{l}\text { Humanos: } \\
\text { - Profesor } \\
\text { - Alumno }\end{array}$ \\
\hline $\begin{array}{l}\text { El profesor revisará } \\
\text { y evaluará el } \\
\text { Trabajo } \\
\text { Especial de Grado } \\
\text { en su conjunto. }\end{array}$ & $\begin{array}{l}\text { Evaluar: } \\
\text { - Estilo } \\
\text { - Forma } \\
\text { - Contenido }\end{array}$ & & $\begin{array}{l}\text { Materiales } \\
\text { - Material } \\
\text { Bibliográfico } \\
\text { - Tabla de } \\
\text { evaluación }\end{array}$ \\
\hline
\end{tabular}




\section{Cuadro № 3}

Plan operativo del objetivo (Seminario II) cont..

\begin{tabular}{|c|c|c|c|}
\hline Objetivo terminales & Actividades & Tiempo & Recursos \\
\hline $\begin{array}{l}\text { El profesor } \\
\text { enseñará a los } \\
\text { Tesistas sobre los } \\
\text { medios } \\
\text { instruccionales a } \\
\text { utilizar en la } \\
\text { exposición y } \\
\text { defensa del T.G }\end{array}$ & $\begin{array}{l}\text { Enseñar la } \\
\text { utilización de los } \\
\text { medios ins- } \\
\text { truccionales: } \\
\text { - Rotafolio } \\
\text { - Retroproyector } \\
\text { - Diapositivas } \\
\text { Concretar con el } \\
\text { Coordinador la } \\
\text { fecha de la defensa } \\
\text { del Trabajo deGrado }\end{array}$ & $\begin{array}{l}3 \text { Unidades créditos } \\
\text { cada seminario con } \\
\text { un total de } 96 \\
\text { horas/ semestre. }\end{array}$ & $\begin{array}{l}\text { Humanos: } \\
\text { - Profesor } \\
\text { - Alumno } \\
\text { Materiales } \\
\text { Recursos } \\
\text { instruccionales } \\
\text { - Retroproyector } \\
\text { - Diapositivas } \\
\text { - Computadoras }\end{array}$ \\
\hline
\end{tabular}

ya sea que se conciba como hechos, o como cuerpos organizados de conocimientos plenos en el área de estudio y a los individuos inmersos en el sistema. Los actores involucrados en el sistema tutorial tienen la responsabilidad de dar cumplimiento al sistema y hacen garantizar la transferencia de conocimiento y de acción (ver gráfico No. 2), para ello es necesario tres tipos de actores: los que tienen capacidad de conducir, los que conducen el proceso y los que tienen gobernabilidad sobre el proceso.

Por lo tanto es indispensable que el programa produzca información, para ello se ha elaborado un Flujograma de Actividades (ver gráfico 3 y 4) que debe realizar el tutor en el proceso de los Seminarios de Trabajos de Grado.

Se recomienda aplicar un Flujograma en la Coordinación de Seminarios a fin de que se haga posible monitorear y evaluar tanto la gestión del tutor como los resultados obtenidos en los diferentes se- minarios acorde a las líneas de Investigación que tenga cada tutor en el proceso que conduce.

\section{Sistema de seguimiento y evaluación del programa}

\section{Objetivo del Sistema}

La finalidad de este Sistema consiste en preesentar una serie de procesos a fin de mantener un seguimiento constante en el desarrollo de los Seminarios de Grado I y II para lograr un reforzamiento académico cada Semestre. Ver gráfico No. 5.

\section{Evaluación de la ejecución supervisora del tutor}

El presente instructivo fue diseñado con base a una serie de razonamientos básicos para desarrollar el proceso de evaluación del Sistema tutorial. Estos ra- 
Gráfico № 1

Organización del sistema

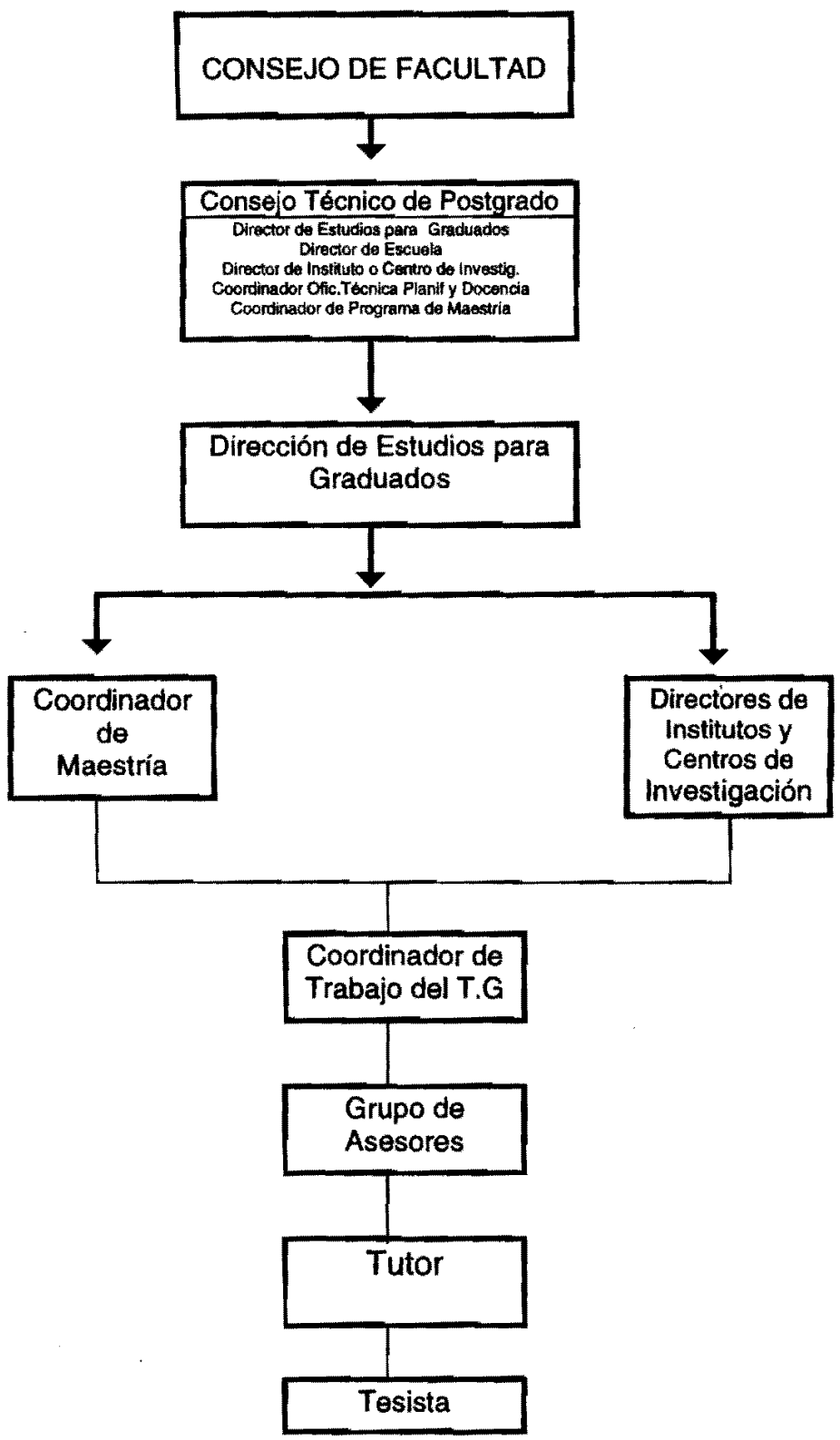


Gráfico №2

Flujograma de información

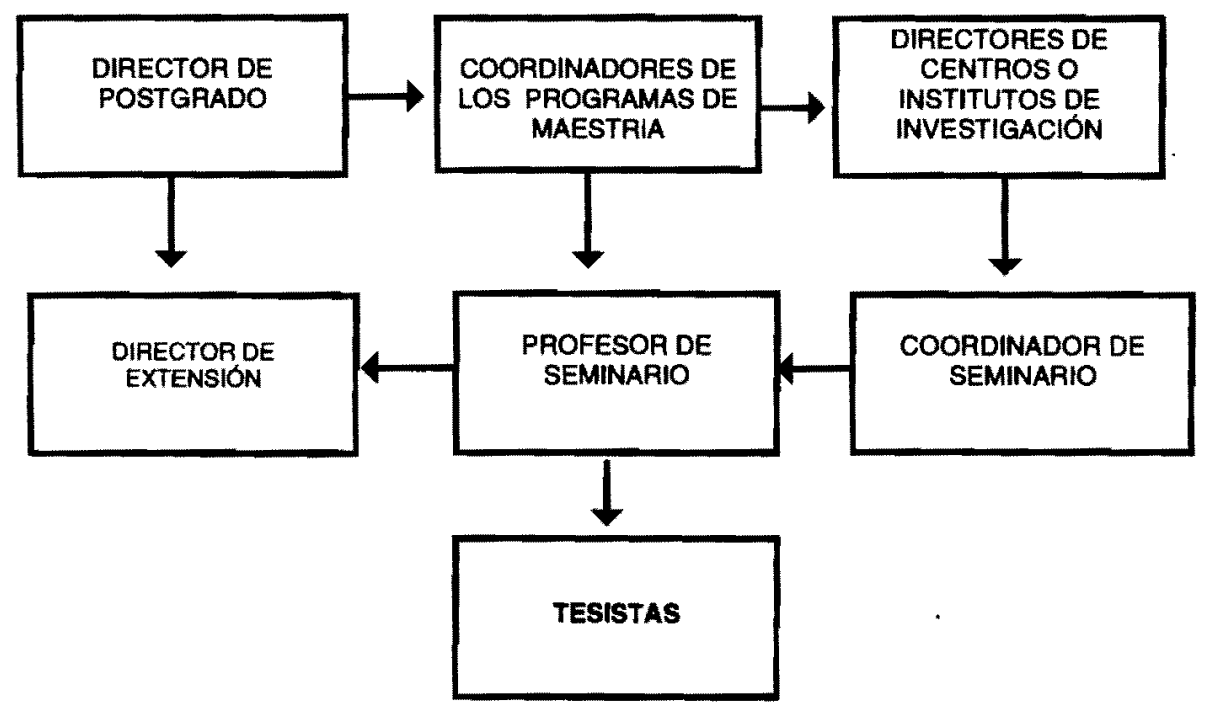




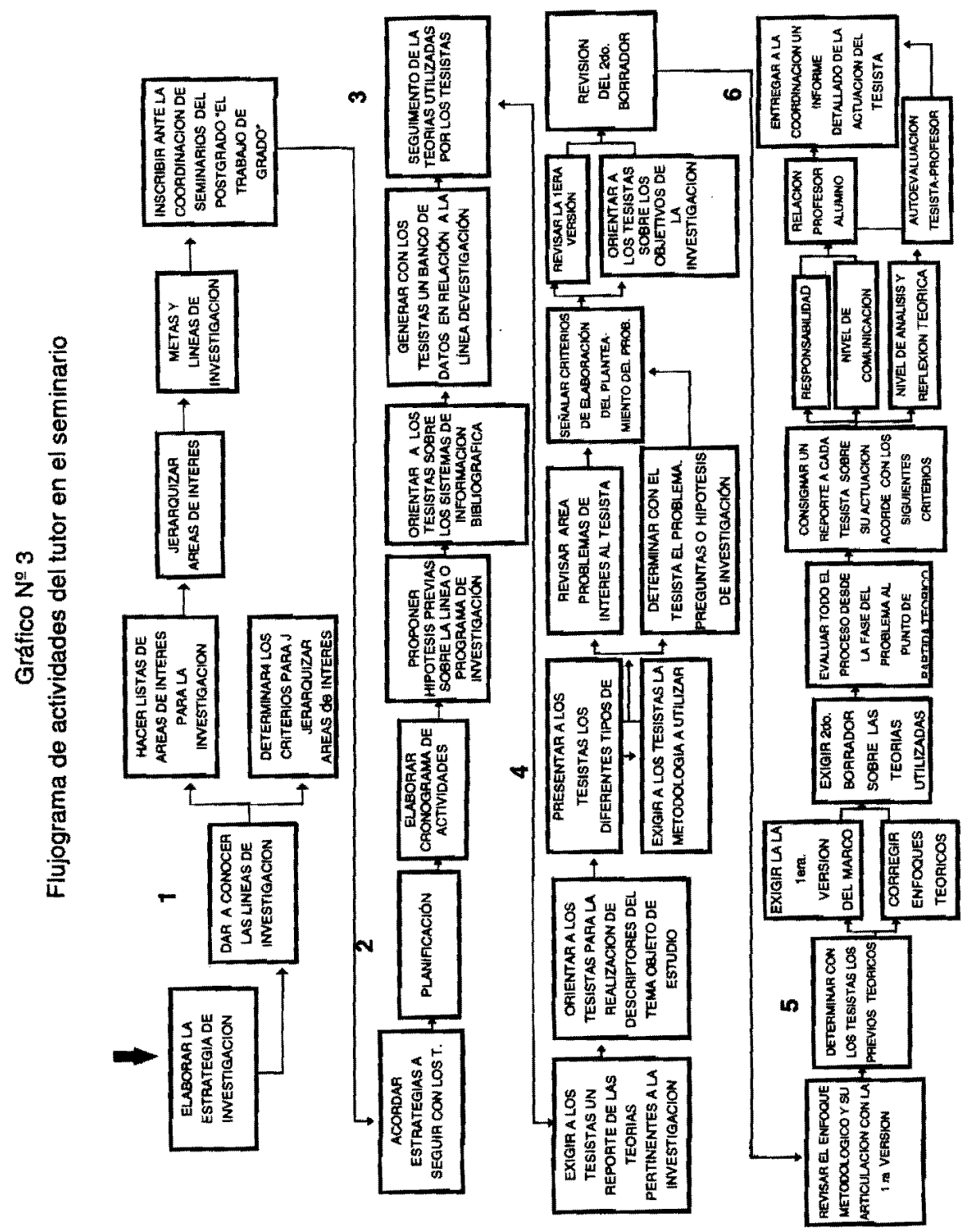




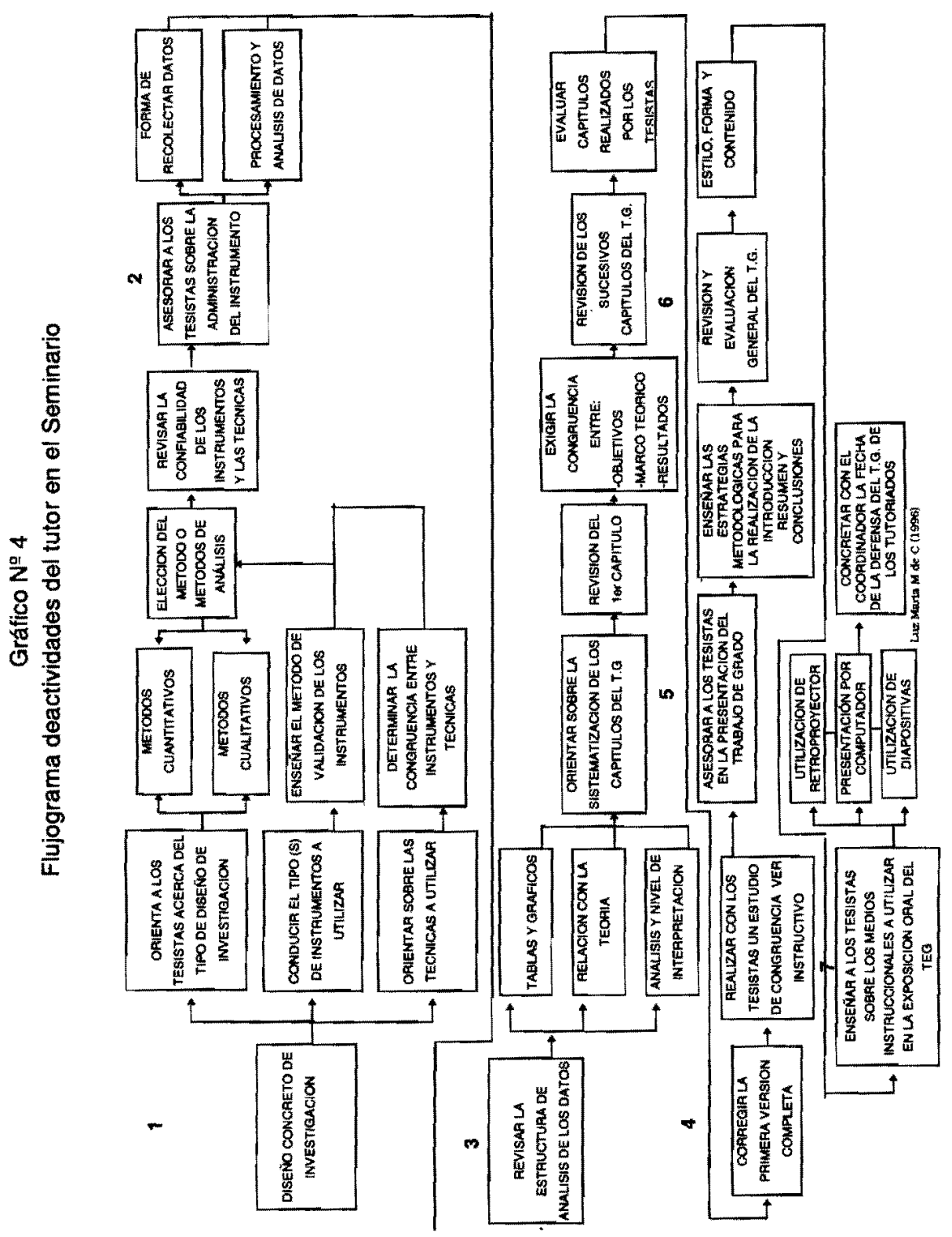




\section{Gráfico № 5}

Esquema general de evaluación



zonamientos se fundamentan en el desenvolvimiento de los roles de los profesores que dictan seminarios, tanto sus expectativas como la ejecución de las actividades asignadas.

Las interrogantes, en que se basa el diseño son las siguientes:

a) Cuáles son las razones para evaluar la ejecución del Sistema Tutorial? ¿Por Qué?

b) Qué aspectos deben ser evaluados en la ejecución del Sistema Tutorial? ¿Qué?

- Actividades académicas

- Habilidades y destreza en el manejo del grupo.

- Responsabilidad - Relaciones interpersonales.

Cómo puede ser evaluado el Sistema Tutorial? ¿Cómo?

c) Esta pregunta abarca los siguientes aspectos:

- Tipo de Evaluación

- Ex-ante
- Formativa

- Ex-post

- Estrategias a desarrollar

- Plan de acción

- Instrumentación

- Evaluación del coordinador

- Autoevaluación del profesor

- Coevaluación (alumnos y profesor)

- Implementación y Validación

- Centro de Validación

d) Cuándo debe ser evaluado el Sistema Tutorial?

- Selección del tiempo más apropiado para ello

- Aplicación de Instrumentos

- Recopilación de Información

e) ¿Quién debe evaluar la ejecución supervisora? ¿Quién?

- Los coordinadores de los Programas de Postgrado son los encargados de llevar a cabo la ejecución del Instrumento.

El análisis de los resultados es uno de los aspectos más importante del pro- 
ceso, ya que servirá de instructivo para cada profesor, de allí se tomarán las previsiones a que dé lugar.

La evaluación de los Tutores a este nivel, puede dar origen al establecimiento de criterios para evaluar el Sistema Tutorial, así como también estimar los alcances del mismo, en lo relativo a los procesos pedagógicos, cognoscitivos y de Investigación que cumple el profesor en cada Seminario.

- Una de las razones poderosas para evaluar el Sistema, consiste en establecer las pautas de la ejecución tutorial, con la finalidad de lograr los objetivos del proceso.

- Otra razón importante, radica en determinar la eficacia de los Tutores en el ejercicio de sus funciones, en ambos seminarios.

A través de esta evaluación, los docentes se percatarán de sus aciertos y limitaciones en su ejecución, al determinar los factores necesarios para lograr una administración efectiva en el desarrollo de las tareas específicas en el proceso tutorial.

\section{Aspectos que deben ser evaluados en el sistema tutorial}

Entre los aspectos que deben ser evaluados en la ejecución supervisora están:

- Habilidades y destrezas

- Control de las destrezas y conocimientos de las tareas asignadas en el proceso tutorial.
- Dominio de los aspectos teóricos, técnicos y prácticos para el funciona. miento de cada seminario.

* Responsabilidad:

- Cumplimiento en el desempeño de las tareas asignadas, iniciativa $y$ efectividad en el proceso.

- Asistencia y puntualidad.

- Actitud asumida ante las obligaciones y situaciones en el trabajo.

* Relaciones Interpersonales:

- Efectividad en los conflictos interpersonales.

- Comunicación verbal y no verbal.

- Consenso ante el grupo que dirige.

- Participación efectiva de todo el personal Profesoral que integra el equipo,

- Cooperación en las situaciones planteadas.

* Cortesía y trato hacia el grupo que dirige.

- Crecimiento profesional:

- Adquisición de nuevos conocimientos

- Disposición para colaborar en el desarrollo de habilidades y destreza de los tesistas.

- Actualización permanente de conocimientos en el campo de la investigación

* Manejo del grupo y utilización de Recursos:

- Eficiencia en la delegación de funciones y responsabilidades con autoridad y dinamismo. 
- Clara definición de patrones de ejecución.

- Definición de políticas dentro de una concepción abierta.

- Manejo de situaciones conflictivas.

* Supervisión y Evaluación del grupo a su cargo:

- Evaluación del grupo de seminario con objetividad.

- Supervisión del grupo.

- Información acerca de la actuación del proceso tutorial.

- Normativa dentro del proceso tutorial.

* Condiciones Personales:

- Auto-control: dominio de si mismoen diversas situaciones.

- Honestidad: Cumplimiento de las tareas asignadas, aceptación de las debilidades y flaquezas que pudieren surgir, exponer con franqueza los puntos de vista, aceptar las críticas negativas, persuadir sobre el buen uso del tiempo.

* Comunicación:

- Habilidad para trabajar en grupos (Departamentos, Secciones y Seminarios).

- Aceptación de nuevas ideas.

- Comunicación oportuna de la información.

* Liderazgo:

- Planificación: trazar planes lógicos de trabajo en el Sistema Tutorial.

- Coordinación: Ordenar las diferentes actividades de trabajo.

- Organización: Utilizar e inducir en forma adecuada el tiempo y los recursos disponibles.

- Control: mecanismos de trabajo que orienten y regulen la calidad y eficiencia del mismo.

\section{Consideraciones finales sobre el sistema de seguimiento}

El objeto primordial de la evaluación del profesor de los Seminarios I y II, consiste en colaborar con el mismo, a fin de que éste reconozca sus deficiencias, y promueva su autocrecimiento: personal y profesional.

- Ofrecerle a los profesores una retroalimentación constante en la ejecución del proceso tutorial para el beneficio personal del grupo que asesora y la dependencia universitaria..

- Lograr con este proceso evaluativo, que los profesores reconozcan sus fallas y exijan a la institución un programa de actualización en aquellas áreas que se relacionen con la acción tutorial.

- Mantener la armonía y entusiasmo con el personal docente, ya que su acción se desplegaría directamente hacia los tesistas y a toda persona involucrada en el proceso que tiene relación con el Trabajo de Grado.

La evaluación es un proceso que permite conocer los recursos humanos de cualquier institución, de sus cualidades profesionales, preparación y rendimiento académico, también permite obtener una visión general del potencial humano con que cuenta la institución. 
De igual forma, permite lograr una administración más racional de las políticas relacionadas con la academia y el personal docente, así como todas aquellas acciones tendientes a satisfacer la efectividad del proceso Tutorial.

* Esta evaluación debe representar una opinión objetiva e imparcial y no permitir que los sentimientos personales y prejuicios influyan en los resultados de este proceso.

- El evaluador debe pensar y analizar con detenimiento cada uno de los aspectos a ser evaluados, a fin de que los criterios priven independientemente unos de otros.

- El coordinador debe discutir con el profesor evaluado, los resultados de la evaluación, a fin de intercambiar impresiones sobre la misma.

Esta evaluación debe ser realizada semestralmente por el Coordinador de Seminarios.

\section{Referencias Bibliográficas}

Brenes, Alban. (1987). "Los Trabajos Finales de Graduación". Su Elaboración y presentación en las Ciencias Socia- les. Edit. Universidad Estatal a Distancia. Costa Rica.

Carrier, Hervé. (1977). "Misión Futura de la Universidad". Edit. El Ateneo Buenos Aires.

Castro, P. Manuel. (1990) "Taller sobre el análisis académico de facilitadores a nivel de Postgrado y Líneas de acción". Universidad Nacional Abierta. Caracas. S/F.

Dressell, Paul. (1976). "Handbook of Academic Evaluation": Assessing Institutional Efectiveness in Higher Education. San Fco. Jossey Bass.

Farmer, Charles H. (1981). "Administrator Evaluation: Concepts, Methods, cases in Higher Education Leadership E. Manage Ment Society, Inc.

Hiliway, Tyrus. (1979), "Evaluating College And University Administration". Jossey - Bass, Traducido por R!chards Piter.

Parsons, T. y Shils, E. (1990) "Hacia una Teoría General de la Acción". Public. Harvard University Press, Cambridge.

Sabino, Carlos A. (1980). "El Proceso de Investigación". El Cid Editor. Argentina.

Consejo Universitario (1994) 